\title{
The Radiation Sensitivity of Normal Mouse Bone Marrow Cells, Determined by Quantitative Marrow Transplantation into Irradiated Mice ${ }^{1}$
}

\author{
E. A. McCULLOCH AND J. E. TILL \\ Department of Medical Biophysics, University of Toronto, and the Divisions of Biological Research \\ and Physics of the Ontario Cancer Institute, Toronto, Ontario
}

INTRODUCTION

The number of viable cells remaining in any sample of irradiated cells is determined by finding how many are capable of continued proliferation. Two methods for accomplishing this have been described, and both are limited in their application. The first method (1) depends on irradiating cells in tissue culture and finding how many can subsequently form clones; this method is open to the objection that cells may behave differently in culture from the way they do in the animal. The second method (2) is limited to tumor cells and depends on ascertaining how many cells of any irradiated sample are capable of developing into tumors after transplantation into new hosts. Neither method permits the determination of the viability, for example, of the cells of normal bone marrow growing in their normal situation after irradiation.

The method described in this paper for determining the number of viable cells in bone marrow irradiated in vivo and in vitro hinges on the fact that marrow cells from normal mice, injected into lethally irradiated mice, multiply and differentiate within the hemopoietic organs of the recipients so as to prevent their death from marrow failure (3-6). Thus the survival of an irradiated mouse depends on the viability of cells in the transplanted marrow. Further, it has been shown that the percentage survival of irradiated animals receiving marrow injections is a function of the total number of cells injected $(7,8)$. Therefore, the percentage survival of a group of irradiated mice injected with bone marrow can be used to measure the number of viable cells in the suspension used for injection. In the experiments

\footnotetext{
1 The research for this paper was supported (in part) by the Defence Research Board of Caiaada, under Grant DRB 9350-14 (G\&C) and (in part) by the National Cancer Institute of Canada.
} 
described below, this method was used to determine the radiation sensitivity of normal bone marrow cells.

\section{EXPERIMENTAL PROCEDURES}

The mice were C57Bl/Ha and Swiss/ICR bred in this laboratory. Each experimental group consisted of 10 males and 10 females, from 2 to 3 months old. Littermates were evenly distributed among the experimental groups.

Twenty-five to thirty mice, half males and half females, but of the same age and strain as the recipients, were used as marrow donors in each experiment. These were killed with ether. Both femora were cleaned of muscle and their ends cut off with scissors. A No. 20 needle was then pushed into the marrow cavity, and icecold balanced salt solution (Puck's saline A) was aspirated backward and forward through the cavity until all the marrow was removed. By this procedure the yield from 30 adult mice was usually 12 to $15 \mathrm{ml}$ of a suspension containing 4 to $8 \times 10^{7}$ nucleated marrow cells per milliliter. Cell counts were made in a hemacytometer after dilution of $0.5 \mathrm{ml}$ of the suspension to $5 \mathrm{ml}$ in acetic acid with added methylene blue. Reproducible counts $( \pm 5 \%)$ were obtained down to concentrations of $10^{6}$ cells $/ \mathrm{ml}$. The original marrow suspension was diluted appropriately in balanced salt solution to give suspensions with the required cell concentration. The sample containing $10^{6}$ cells $/ \mathrm{ml}$ was always checked by direct count. During all manipulations, the marrow suspensions were kept at ice-water temperature.

The mice to be injected with bone marrow cells ("recipient mice") were irradiated in groups of 10 in a circular Lucite box on a revolving turntable $50 \mathrm{~cm}$ from the target of a 280-kvp X-ray generator, operated at 20-ma tube current, with filtration giving a HVL of $1.12 \mathrm{~mm} \mathrm{Cu}$. The exposure dose rate at $50 \mathrm{~cm}$, including scattered radiation, was $160 \mathrm{r} / \mathrm{min}$. Ionization measurements were made with a Philips universal dosimeter and a Victoreen 100-r chamber, both calibrated against a Victoreen 25-r chamber calibrated by the U. S. National Bureau of Standards. Routinely, the absorbed dose received by the soft tissues of the recipient mice was 950 rads, with a variation in dose through the mouse of between 10 and $15 \%$. This dose, as will be shown (Fig. 1), was above that necessary to cause $100 \%$ mortality of untreated mice in 30 days.

Marrow suspensions were irradiated in 12-ml polyethylene centrifuge tubes. Chilled 6-ml samples were agitated immediately before irradiation to ensure that the cells were in suspension and adequately aerated. The tubes were placed in a rotating Styrofoam tube holder and irradiated with 280-kvp X-rays, with HVL of $1.12 \mathrm{~mm} \mathrm{Cu}$, at an average exposure dose rate of $115 \mathrm{r} / \mathrm{min}$. Simultaneously, ionization measurements were made with a 100-r Victoreen chamber.

As soon as possible after irradiation the recipient mice were given $0.5 \mathrm{ml}$ of bone marrow suspension injected intravenously into the prewarmed tail vein. Though sometimes an animal died of suffocation or air embolism during the procedure, no experimental group was reduced to less than 18 mice because of accidental deaths. 
Frequently, at the end of the injections, counts were made on the remaining suspension. These agreed well with those done at the beginning of the experiment, indicating that adequate mixing had been done during the period of the injections.

After these procedures the mice were housed 3 to 4 animals to a cage and allowed food (Purina Mills mouse diet) and water freely. The cages were examined at least once a day, and the dead animals removed. In this way the 30-day survival of each experimental group was determined.

For the in vivo irradiation of marrow, various doses of $\mathrm{X}$-ray were given to groups of 10 mice by the method already described. The marrow was then extracted from the femurs of the mice of each group, cell counts were made, and the suspension was diluted to a cell count of $10^{6}$ cells per $0.5 \mathrm{ml}$. This amount of each suspension was then injected intravenously into each of a group of 20 mice that had received the standard dose of 950 rads. The recipients were then followed in the usual manner.

\section{RESULTS}

\section{X-Ray Mortality of Swiss Mice}

Mice to be used as recipients of bone marrow received an absorbed X-ray dose in their soft tissues of 950 rads. This dose was chosen on the basis of experiments in

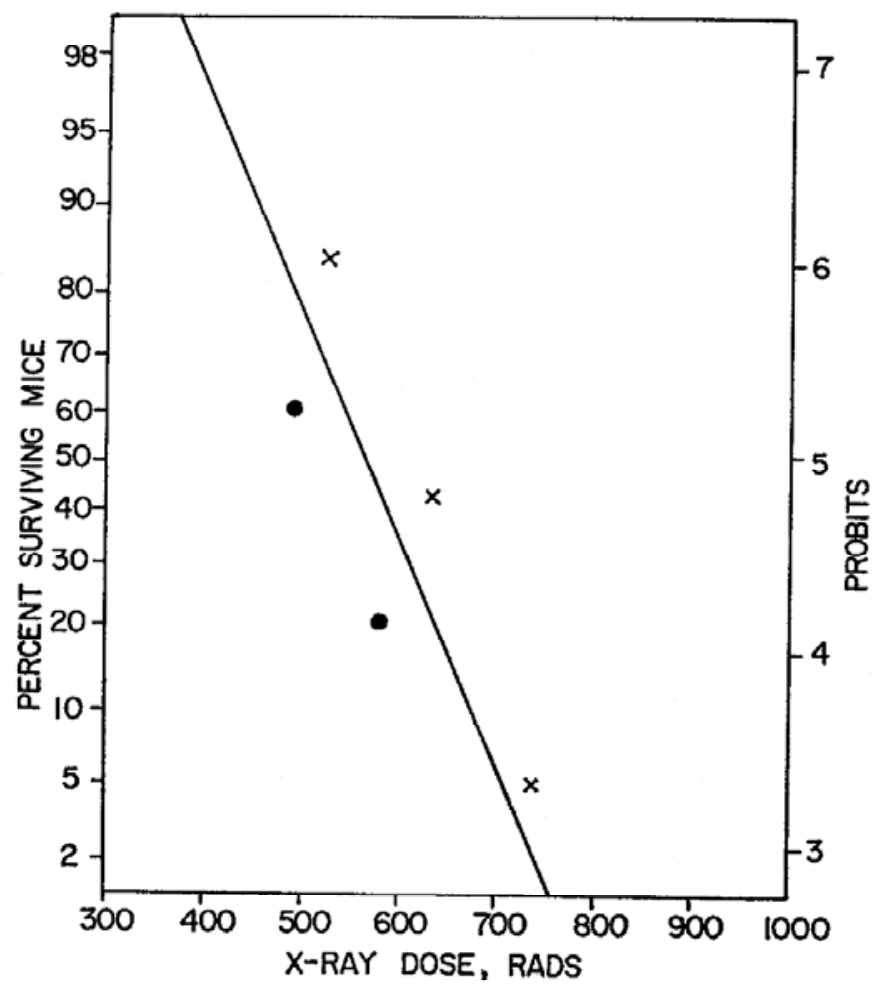

FIG. 1. Probit plot of the survival curve for Swiss mice given various doses of wholebody X-irradiation. 


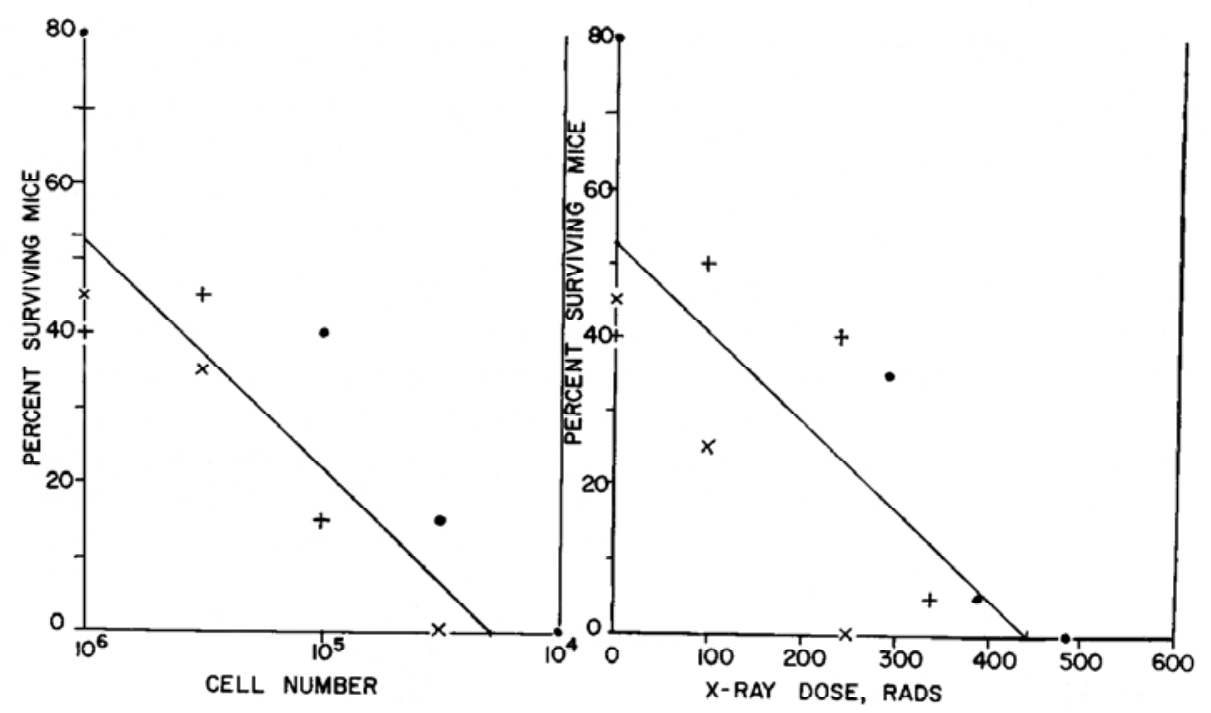

FIG. 2. A, survival of groups of lethally irradiated Swiss mice plotted against the number of cells injected intravenously after irradiation. Results of three experiments, with each experiment represented by a separate symbol. $B$, survival of groups of lethally irradiated Swiss mice injected with marrow cells irradiated in vitro, plotted against the dose of radiation given the cells. The aliquots were from the same experiments the results of which are shown in Fig. $2 A$.

which groups of 20 Swiss mice were given various X-ray doses without subsequent treatment. Figure 1 shows the results of two such experiments on a probit plot. These results indicate that there was a vanishingly small probability of any mouse's surviving a dose of 950 rads. Similar dosage experiments were not done for the C57Bl strain, but there were no survivors in four groups of 20 mice of this strain given this dose followed either by no treatment or by the injection of $10^{4}$ marrow cells or less. In addition, many authors have reported experiments in which doses of the order of 950 rads were shown to be in the supralethal range (9).

\section{Mortality of Irradiated Recipient Swiss Mice Injected with Various Concentra- tions of Unirradiated Bone Marrow}

The reduction in mortality of recipient Swiss mice irradiated with 950 rads of Xrays, as a function of the number of marrow cells injected, was determined by using dilutions of a single marrow suspension. The results of three separate experiments are shown in Fig. 2A. Each point represents the percentage of a group of 20 recipient mice still alive 30 days after irradiation. The results indicate a progressive increase in the percentage of surviving mice as the number of marrow cells injected is increased from $10^{4}$ to $10^{6}$ cells per recipient mouse. For cell numbers above $10^{6}$, 
little further enhancement of survival of the recipient mice occurs. The straight line shown in Fig. $2 A$ is a least-squares fit to the points from the three experiments, on the assumption that there is a linear relationship between percentage survival and log cell number.

\section{Mortality of Recipient Swiss Mice after Injection of Irradiated Marrow}

Aliquots containing $10^{6}$ cells from the same marrow suspensions that were used in the dilution experiments described above were irradiated in vitro with various $\mathrm{X}$-ray doses and injected into irradiated recipient mice. The irradiation acts like a dilution of viable cells in that it reduces their numbers in the suspension. As expected, the percentage of irradiated recipient mice surviving after being injected with irradiated bone marrow decreased as the dose to the marrow was increased, as shown in Fig. 2B. The straight line shown is a least-squares fit to the data. To construct a survival curve for bone marrow cells from Fig. $2 A$ and $2 B$, it is only necessary to assume that, for a given level of survival conferred on the irradiated recipient mice, the same number of viable cells is present in both the irradiated and the
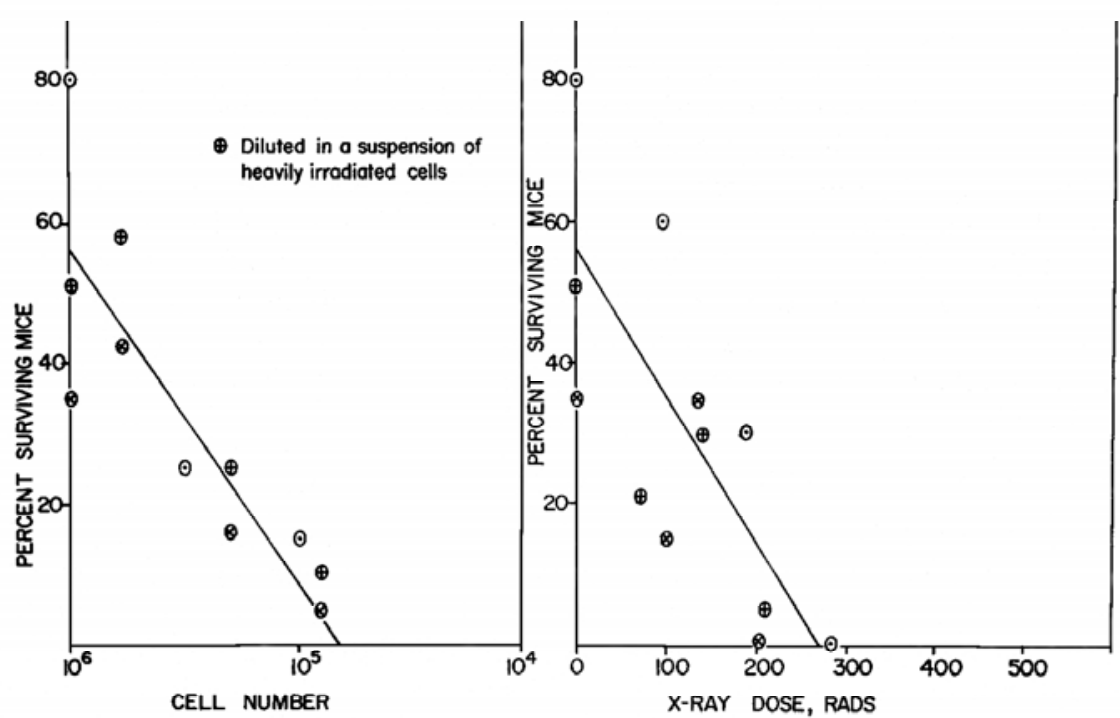

FIG. 3. A, survival of groups of lethally irradiated C57Bl mice plotted against the number of cells injected intravenously after irradiation. Result of three experiments, with each experiment represented by a separate symbol. B, survival of groups of lethally irradiated C57Bl mice injected with marrow cells irradiated in vitro, plotted against the dose of radiation given the cells. The aliquots were from the same experiments the results of which are shown in Fig. $3 A$. 
diluted suspensions, as will be discussed in Section 6. That this survival curve is not limited to Swiss mice is shown by the results of experiments with $\mathrm{C} 57 \mathrm{Bl}$ mice. These results will be given in the next section.

\section{Dilution and Irradiation of C57Bl Marrow .}

Suspensions of $\mathrm{C} 57 \mathrm{Bl}$ marrow cells were injected intravenously into $\mathrm{C} 57 \mathrm{Bl}$ recipients in three experiments similar to those done with Swiss mice. The results are shown in Fig. $3 A$, in which the percentage of surviving mice is plotted against the number of cells injected, and in Fig. 3B, where the percentage of surviving mice is plotted against the dose of $\mathrm{X}$-rays to the marrow suspension in the same manner as in Figs. $2 A$ and $2 B$. The effects of dilution and irradiation on the ability of suspensions of $\mathrm{C} 57 \mathrm{Bl}$ marrow cells to enhance survival when injected into irradiated isologous mice are of the same order as those observed for suspensions of Swiss mouse marrow cells.

\section{Dilution of C57BI Marrow in a Suspension of Heavily Irradiated Cells}

To test for any possible influence exerted by radiation-damaged cells in the suspensions to be injected, a reconstruction experiment was performed. In one of the C57BI experiments an aliquot of a marrow suspension prepared in the usual way was given an X-ray dose of 5000 rads in order to inactivate the cells. This suspension of heavily irradiated cells was then used as a diluent in preparing the marrow dilutions, so that in the dilution experiment each aliquot contained $10^{6}$ heavily irradiated cells in addition to the known number of viable cells. The results of the reconstruction experiment are included in Fig. $3 A$ and are not different from those of the other two experiments in which the cells were diluted in balanced salt solution, showing that the presence of radiation-damaged cells in the suspension does not affect the ability of viable marrow cells to enhance the survival of the irradiated recipient mice.

\section{The X-Ray Sensitivity of Normal Mouse Bone Marrow Cells}

The results of the experiments on dilution and irradiation of Swiss and C57Bl marrow cells were used to construct an X-ray survival curve for mouse bone marrow cells in the following way: The least-squares straight lines drawn through the data of Figs. $2 A$ and $3 A$, representing the percentage of irradiated recipient mice surviving after injection of various numbers of cells, were used as "calibration curves" to determine the number of viable cells present in the suspensions of marrow cells given different doses of $\mathrm{X}$-rays. The way in which this is done can best be illustrated by means of an example: One of the suspensions of C57Bl marrow cells was given a dose of 215 rads of radiation. On injection of this irradiated marrow suspension into irradiated recipient mice, $5 \%$ of the injected mice were alive after 30 days. This is the percentage of mice that normally survive after the injection of $8.5 \times 10^{4}$ 


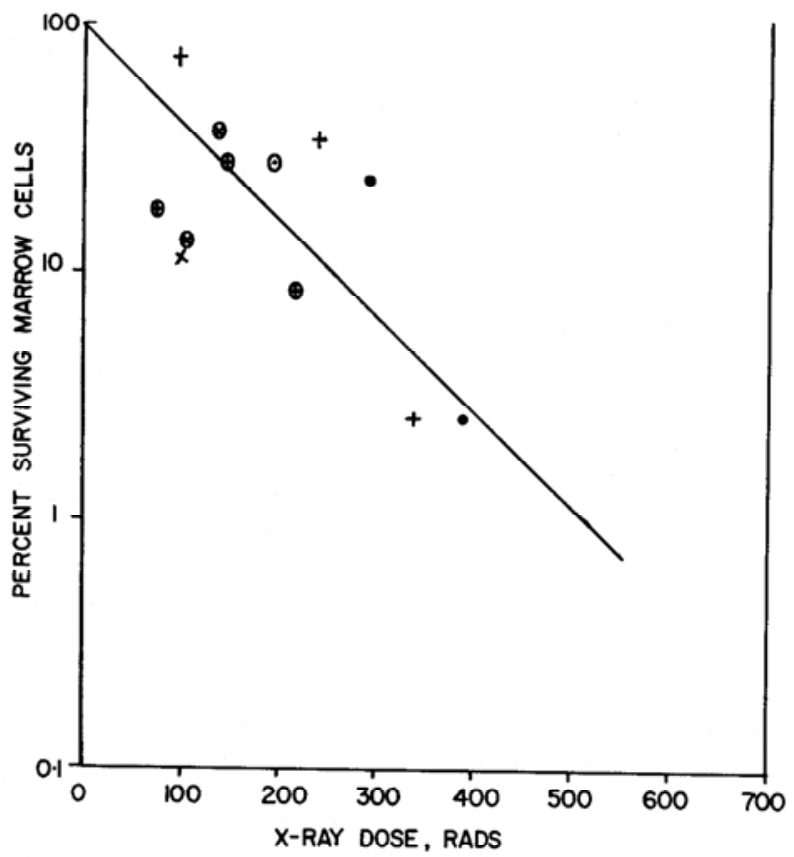

FIG. 4. X-Ray survival curve for normal mouse marrow. The symbols are those of the experimental groups (Figs. 2 and 3) from which the points were obtained.

unirradiated $\mathrm{C} 57 \mathrm{Bl}$ marrow cells, as may be seen from Fig. 3A. It is therefore assumed that the dose of 215 rads reduced the number of viable cells in the irradiated marrow suspension from its initial value of $10^{6}$ to $8.5 \times 10^{4}$, i.e., that the percentage survival of the irradiated marrow cells was $8.5 \%$. The results shown in Fig. 4 were obtained by treating the data of Figs. $2 B$ and $3 B$ in this way.

The method is not sufficiently sensitive to allow a description of the form of the survival curve, and a simple exponential relationship was assumed. When a leastsquares straight line was fitted to the data, a value of 105 rads was found for the dose required to reduce cell survival to $37 \%$ of its initial value $\left(D_{37}\right)$.

The $D_{37}$ may be obtained without constructing the survival curve shown in Fig. 4 . It may be shown readily that the $\mathrm{D}_{37}$ is given by the following equation:

$$
D_{37}=-0.4318 b_{d} / b_{x}
$$

where $b_{d}$ is the slope of the marrow dilution curve (Figs. $2 A$ and $3 A$ ) and $b_{x}$ is the slope of the marrow radiation curve (Figs. $2 B$ and $3 B$ ). This expression yields a $D_{37}$ 


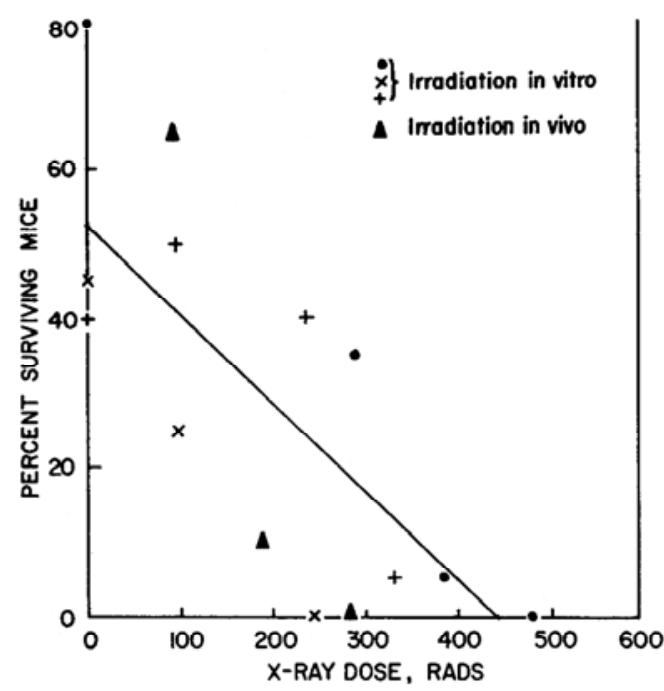

FIG. 5. Comparison of in vivo and in vitro irradiation of marrow. The solid triangles represent the survival of groups of mice that received marrow cells irradiated in vivo. The other points are the same as in Fig. $2 B$.

of $112 \pm 38$ rads for Swiss marrow and $97 \pm 32$ rads for C57Bl marrow. These values do not differ significantly $(\mathrm{P}>0.7)$. Combining the two values yields a mean $\mathrm{D}_{37}$ of $105 \pm 24$ rads, in agreement with the value obtained from Fig. 4. The errors quoted are standard errors, derived from the standard errors of the leastsquares slopes.

\section{In Vivo Irradiation of Marrow}

It is not possible by this technique to compare aliquots of the same marrow suspension irradiated with different doses in vivo. However, the results of the various experiments on the irradiation of marrow in vitro were sufficiently similar to make it reasonable to compare different marrow suspensions at various X-ray doses, provided each contained the same number of nucleated cells. Accordingly, suspensions obtained from groups of 10 Swiss mice irradiated while alive with 96, 192, and 288 rads, respectively, were tested for their ability to prevent death in Swiss mice given 950 rads. The results are given in Fig. 5. The solid line in the figure is taken from Fig. $2 \mathrm{~B}$ and represents the results of irradiating Swiss marrow in vitro. The three points obtained from the groups that received marrow irradiated in vivo are not significantly different from those obtained for marrow irradiated in vitro $(\mathrm{P}>0.2)$. 


\section{DISCUSSION}

Two successful methods of obtaining survival curves for mammalian cells have been described previously. One is the clone-counting technique using cells cultivated in vitro on a glass surface, utilized for both normal and malignant human cells by Puck and Marcus $(1,10)$. This technique yields a value of between 100 and $150 \mathrm{r}$ for the dose of 230-kvp X-rays required to reduce the level of survival to 37 $\%$ of its initial value $\left(D_{37}\right)$. Though the technique is an elegant one, yielding readily reproducible results, it has not yet been demonstrated that a particular cell type shows the same radiation sensitivity when cultivated in vitro as it does in vivo.

A second technique utilizes the transplantation in vivo of malignant cells capable of regenerating tumors from a very small number of cells. This method, applied to mouse lymphoma cells by Hewitt and Wilson (2), yielded a value of $162 \mathrm{r}$ of $\mathrm{Co}^{60}$ radiation for the $\mathrm{D}_{37}$. Since the relative biological efficiency of $\mathrm{Co}^{60}$ radiation compared to 200-kvp X-rays is approximately $0.85(11,12)$, the equivalent $\mathrm{D}_{37}$ of $\mathrm{X}$ rays for the mouse lymphoma cells should be about $15 \%$ less than $162 \mathrm{r}$, i.e., about $140 \mathrm{r}$. Thus, the values for the $\mathrm{D}_{37}$ of human cells in tissue culture and of mouse lymphoma cells are similar.

The experiments described in the present paper provide an estimate of the radiation sensitivity of normal cells from bone marrow growing in vivo after irradiation. The experiments are based on the fact that marrow cells injected intravenously into lethally irradiated animals will permit the survival of a proportion of the recipient animals and that the decrease in mortality is a function of the number of cells given $(7,8)$. Since it has been shown that the injected marrow cells prevent death by repopulating the hematopoietic organs of the recipients, it is possible to use the protective property of marrow as a measure of its content of living cells. Smith (13) has already used this concept qualitatively in experiments on the protective effect of AET given before irradiation. In the experiments reported here, an attempt was made to apply the technique quantitatively. Reasonably reproducible results were obtained even though the cell counts on which the results depend were counts of all the nucleated cells in the marrow suspension. It is not known which cell type or types are capable of multiplication when transplanted into a new animal, but it is unlikely that fully differentiated cells have this capacity. Therefore, in all our experiments the effective cells must have been present as a constant proportion of the total number of nucleated cells.

Further, the transplanted cells not only must divide and form clones but also must possess the capacity to differentiate into functional marrow in order to prevent death of irradiated recipients. Thus the test of viability in our experiments is an unusually stringent one; this may result in an overestimation of the radiation sensitivity of the bone marrow cells.

The experimental results indicate that the radiation sensitivity of normal Swiss 
or $\mathrm{C} 57 \mathrm{Bl}$ marrow cells, expressed as the dose required to reduce the number of viable cells to $37 \%\left(D_{37}\right)$, is $105 \pm 24$ rads, ${ }^{2}$ and that the cells have a similar sensitivity when irradiated in vivo or in vitro. This conclusion is strengthened by a consideration of the survival curve for mice given whole-body radiation (Fig. 1). This curve, though obtained independently, is related to the survival curve for mouse marrow cells (Fig. 4) because, over the dose range covered in the mouse survival curve (Fig. 1), transplantation of isologous marrow will prevent death, indicating that marrow failure is the principle cause of death in untreated animals. The mouse survival curve indicates that deaths begin to occur when 400 rads are given, and that mortality is essentially complete when a dose of 800 rads is reached, a range of 400 rads. From the cell survival curve (Fig. 4) it may be seen that 400 rads will reduce a marrow population to I \% of its original number. From the dilution curve for Swiss mouse marrow (Fig. $2 A$ ) it is apparent that the $10^{6}$ cells allow most of the mice to survive, whereas I \% of that number, $10^{4}$ cells, allows no mice to survive. Thus, the range of 400 rads for the mouse survival curve, in which death results from the inactivation of marrow cells by irradiation in vivo, is consistent with the estimate of the radiation sensitivity of marrow cells obtained from the transplantation experiments.

The similarity between the radiation sensitivities of human cells in tissue culture and mouse lymphoma cells transplanted in vivo suggested the possibility of a characteristic radiation sensitivity for mammalian cells. This hypothesis receives support from the estimate presented here for the radiation sensitivity of normal mouse marrow cells cultivated in vivo after irradiation either in vivo or in vitro.

\section{SUMMARY}

1. A technique for measuring the number of viable cells in a suspension of bone marrow by quantitative transplantation into supralethally irradiated mice has been described.

2. The technique was used to measure the radiation sensitivity of normal mouse bone marrow cells and yielded a result of $105 \pm 24$ rads as the $D_{37}$ for marrow cells.

\section{ACKNOWLEDGMENTS}

The authors wish to thank Mr. David Hall and Mrs. Sheila Zeldin for technical assistance.

RECEIVED: October 23, 1959

\section{REFERENCES}

1. T. T. PUCK and P.I. MARCUS, Action of X-rays on mammalian cells. J. Exptl. Med. 103, 653-666 (1956).

2. H. B. HEWITT and C. W. WILsON, A survival curve for mammalian leukaemia cells irradiated in vivo (implications for the treatment of mouse leukaemia by whole-body irradiation). Brit. J. Cancer 13, 69-75 (1959).

${ }^{2}$ One roentgen of 280-kv X-radiation, HVL $1.6 \mathrm{~mm} \mathrm{Cu}$, is equal to $0.96 \mathrm{rad}$ in soft tissue (14). 
3. E. LORENZ, D. UPHOFF, T. R. REID, and E. SHELTON, Modification of irradiation injury in mice and guinea pigs by bone marrow injections. J. Natl. Cancer Inst. 12, 197-201 (1951).

4. C. E. FORD, J. L. HAMERTON, D. W. H. BARNES, and J. F. LOUTIT, Cytological identification of radiation chimaeras. Nature 177, 452-454 (1956).

5. P. C. NOWELL, L. J. COLE, J. G. HABERMEYER, and P. L. ROAN, Growth and continued function of rat marrow cells in X-radiated mice. Cancer Research 16, 258-261 (1956).

6. D. L. LiNDSLEY, T. T ODELL, JR., and F. G. TAUSCHE. Implantation of functional erythropoictic elements following whole body irradiation. Proc. Soc. Exptl. Biol. Med. 90, 512-515 (1955).

7. L. 0. JACOBSON, E. K. MARKS, and E. 0. GASTON, Observations on the effect of spleen-shielding and the injection of cell suspensions on survival following irradiation. In Radiobiology Symposium, 1954, (Z. M. Bacq and P. Alexander, eds.), pp. 122-133, Academic Press, New York, 1955.

8. P. URSO and C. C. CONGDON, The effect of the amount of isologous bone marrow injected on the recovery of haematopoetic organs, survival and body weight after lethal irradiation injury in mice. Blood 12, 251-260 (1957).

9. C. C. CONGDON, Recovery from radiation injury, with special consideration of the use of bone marrow transplantation. In Progress in Hematology (L. M. Tocantins, ed.), Vol. II, pp. 21-46, Grune and Stratton, New York, 1959.

10. T. T. PUCK, D. MORKOVIN, P. 1. MARCUS, and S. J. CIECIURA, Action of X-rays on mammalian cells. II Survival curves of cells from normal human tissues. J. Exptl. Med. 106, 485-500 (1957).

11. W. K. SiNCLAIR, S. E. GUNTER, and A. COLE. The relative biological effectiveness of 200-kvp Xrays, cobalt-60 gamma-rays, and 22 Mevp X-rays, determined from the dose survival curve of Saccharomyces cerevisiae. Radiation Research 10, 418-432 (1959).

12. J. R. CUnNingham, J. E. TILL, and T. KRUCK, The relative biological effectiveness of 250-kvp Xrays, $\mathrm{Co}^{60}$ gamma rays, and 22-Mev betatron X-rays. Radiation Research 11, 439 (1959).

13. L. H. SMITH, Protective effect of 2-mercaptoethylguanidine on bone marrow cells X-irradiated in vitro. Exptl. Cell Research 13, 627-630 (1957).

14. Report of the International Commission on Radiological Units and Measurements (ICRU) 1956. Natl. Bur. Standards (U.S.) Handbook No. 62, (1957). 